\title{
A systematic review for the antidepressant effects of sleep deprivation with repetitive transcranial magnetic stimulation
}

Qing Tang ${ }^{1}$, Guangming Li ${ }^{2}$, Anguo Wang ${ }^{3}$, Tao Liư ${ }^{4}$, Shenggang Feng ${ }^{5}$, Zhiwei Guo ${ }^{1}$, Huaping Chen ${ }^{1}$, Bin He ${ }^{1}$, Morgan A. McClure ${ }^{1}$, Jun Ou${ }^{1}$, Guoqiang Xing ${ }^{6}$ and Qiwen $\mathrm{Mu}^{1,7^{*}}$

\begin{abstract}
Background: Sleep deprivation (SD) and repetitive transcranial magnetic stimulation (rTMS) have been commonly used to treat depression. Recent studies suggest that co-therapy with rTMS and SD may produce better therapeutic effects than either therapy alone. Therefore, this study was to review the current findings to determine if rTMS can augment the therapeutic effects of SD on depression.

Methods: Embase, JSTOR, Medline, PubMed, ScienceDirect, and the Cochrane Central Register of Controlled Trials were searched for clinical studies published between January 1985 and March 2015 using the search term "rTMS/repetitive transcranial magnetic stimulation AND sleep deprivation AND depress*". Only randomized and sham-controlled trials (RCTs) involving the combined use of rTMS and SD in depression patients were included in this systematic review. The scores of the Hamilton Rating Scale for Depression were extracted as primary outcome measures.

Results: Three RCTs with 72 patients that met the inclusion criteria were included for the systematic review. One of the trials reported skewed data and was described alone. The other two studies, which involved 30 patients in the experimental group (SD + active rTMS) and 22 patients in the control group (SD + sham rTMS), reported normally distributed data. The primary outcome measures showed different results among the three publications: two of which showed great difference between the experimental and the control subjects, and the other one showed non-significant antidepressant effect of rTMS on SD. In addition, two of the included studies reported secondary outcome measures with Clinical Global Impression Rating Scale and a self-reported well-being scale which presented good improvement for the depressive patients in the experiment group when compared with the control. The follow-up assessments in two studies indicated maintained results with the immediate measurements.
\end{abstract}

Conclusions: From this study, an overview of the publications concerning the combined use of rTMS and SD is presented, which provides a direction for future research of therapies for depression. More studies are needed to confirm whether there is an augmentative antidepressant effect of rTMS on SD.

Keywords: Depression, Sleep deprivation, Repetitive transcranial magnetic stimulation (rTMS), Systematic review

\footnotetext{
* Correspondence: muqiwen99@yahoo.com

'Department of Radiology \& Imaging Institute of Rehabilitation and

Development of Brain Function, North Sichuan Medical University Nanchong

Central Hospital, 97 South Renmin Road, Shunqing District, Nanchong

637000, Sichuan, China

7Peking University Third Hospital, 49 Garden North Road, Haidian District,

Beijing 100080, China

Full list of author information is available at the end of the article
}

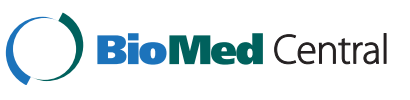

(c) 2015 Tang et al. Open Access This article is distributed under the terms of the Creative Commons Attribution 4.0 International License (http://creativecommons.org/licenses/by/4.0/), which permits unrestricted use, distribution, and reproduction in any medium, provided you give appropriate credit to the original author(s) and the source, provide a link to the Creative Commons license, and indicate if changes were made. The Creative Commons Public Domain Dedication waiver (http://creativecommons.org/publicdomain/zero/1.0/) applies to the data made available in this article, unless otherwise stated. 


\section{Background}

Depression is a major psychiatric disorder that affects people of all age groups. According to the World Health Organization [1], more than 350 million people suffer from depression worldwide. People with depression have a poor quality of life with limited social and occupational functions, which causes enormous social and economic burdens to the patients' families and to society. In addition, the suicide rate of refractory depression patients is high (approximately $15 \%$ ) [2]. The treatment choices for depression include multiple options, such as pharmacotherapy, psychotherapy, electroconvulsive therapy, etc. These therapies can be used alone or in combination. However, not all depressed patients respond equally well to these therapies. For the majority of patients, depression remains intractable. In fact, there is currently neither a complete cure for depression nor sufficient understanding of the complex pathological mechanism underlying depression at present.

Sleep deprivation (SD) has been applied to treat depression since the 1970s [3]. SD is a powerful antidepressant strategy, and it works by depriving the patients of normal sleep, either totally or partially. Research has shown that SD produces a marked effect within hours in 40 to $60 \%$ of depressed patients [4]. Now, SD has become a common protocol for treating depression. In the recent fMRI study reported by Bosch et al. [5], SD reduced functional connectivity between the posterior cingulate cortex and the bilateral anterior cingulate cortex but enhanced connectivity between the dorsal nexus and the distinct areas in right dorsolateral prefrontal cortex (DLPFC). Moreover, other studies showed that SD was associated with altered neurotransmitter receptor sensitivity and neuroendocrine reactivity [6-8].

In spite of its fast therapeutic effect on depression, SD therapy alone has limitations. The duration of SD's therapeutic effect is often short-lived, and the depressive symptoms may return soon after the SD treatment is stopped. Hemmeter et al. [4] showed that more than $80 \%$ of the patients who responded to SD relapsed into depression after one night of recovery. In light of its transient effect, different strategies have been tested to augment and prolong the antidepressant effects of SD. Benedetti et al. [9] reported that lithium carbonate could prolong the antidepressant effect of SD. Wu et al. [10] reported that the addition of three non-invasive, circadian-related interventions to SD therapy in bipolar patients who had been medicated could accelerate and maintain the antidepressant response which provided a quick, safe, and sustainable treatment strategy.

Transcranial magnetic stimulation (TMS) is a newly developed non-invasive therapy that was first reported by Barker et al. in 1985 [11, 12]. TMS causes depolarization or hyperpolarization of neurons in the brain. Soon after its invention, a more advanced form of TMS, i.e., repetitive TMS (rTMS), was developed as a treatment for various neurological and psychiatric disorders, including depression, Parkinson's disease, stroke, and tinnitus. Numerous clinical trials of rTMS therapy have been conducted in depressive patients. Certain types of rTMS with specific parameters have been effective for depressive patients [13-22]. In fact, rTMS was approved in 2008 as a therapeutic option for treatment-resistant major depressive disorders by the U.S. Food and Drug Administration [23]. Although the mechanism is still unknown, rTMS is thought to exert its therapeutic effect on depression by influencing subgenual anterior cingulate functional connectivity $[24,25]$, brain networks [25], neurotransmitters [26], neuroendocrine effects [27], and neuronal plasticity [28].

Researchers have shown that rTMS can augment or sustain the therapeutic effects of SD in patients with depression, although it has not yet been firmly established how rTMS exerts its augmenting effect on SD therapy. Krstić and Ilić [29] reported that a combination of slow-rate rTMS and partial SD (PSD) had strong synergistic effects on depression. Several randomized and sham-controlled trials (RCTs) have investigated the contributions of rTMS to SD treatment in depression with varying outcomes [30-34]. However, single trials alone usually lack persuasive power due to small sample sizes. Because guidance is needed for the clinical application of rTMS-SD co-therapy, a detailed analysis of the current findings is highly desirable. The goal of this study was to systematically review and analyze the published RCTs involving rTMS-SD co-therapy for patients with depression.

\section{Methods}

This systematic review of rTMS and SD co-therapy for depression was conducted in accordance with the Preferred Reporting Items for Systematic Reviews and Meta-Analyses statement (Additional file 1) as well as with the explanation and elaboration document that evaluates health care interventions [35-37].

\section{Search strategy}

Embase, JSTOR, Medline, PubMed, ScienceDirect, and the Cochrane Central Register of Controlled Trials were searched to identify relevant studies. The search term was "rTMS/repetitive transcranial magnetic stimulation AND sleep deprivation AND depress"*. Because rTMS was first proposed in 1985, the searches were limited to human studies published between January 1985 and March 2015. There 
was no restriction with respect to language in the searches.

\section{Study selection}

The full text of the articles that appeared to be relevant was carefully checked by two reviewers independently. Publications that met the following criteria were included in the systematic review [38]:

(1)The study was an RCT, and the number of participants was no less than five in each arm.

(2)The subjects were between 18 and 75 years of age and were diagnosed as having major depression, bipolar disorder, or another form of depression according to the Diagnostic and Statistical Manual of Mental Disorders, 4th Edition (DSM-IV) [39] or Mini-International Neuropsychiatric Interview Criteria (MINI) [40], without any pseudo-depression, history of hysteria or mania, alcoholics, adreno-cortico- tropic-hormone abnormality, hormonal impairment, congenital anomalies, or intake of any anti-depressants.

(3) The treatment protocol was that the subjects received active or sham rTMS over the left or right DLPFC as an added treatment to SD.

(4) The outcome measures were reported (continuous scales) and evaluated with the Hamilton Rating Scale for Depression (HRSD) [41], Clinical Global Impression Rating Scale (CGI) [42], MontgomeryÅsberg Depression Scale (MADRS) [43], or other scales for depression.

Studies were excluded if they had the following characteristics:

(1)The study enrolled subjects who also had other severe neurologic or psychiatric diseases, who had a history of seizures or brain surgery, or who were implanted with electronic devices.
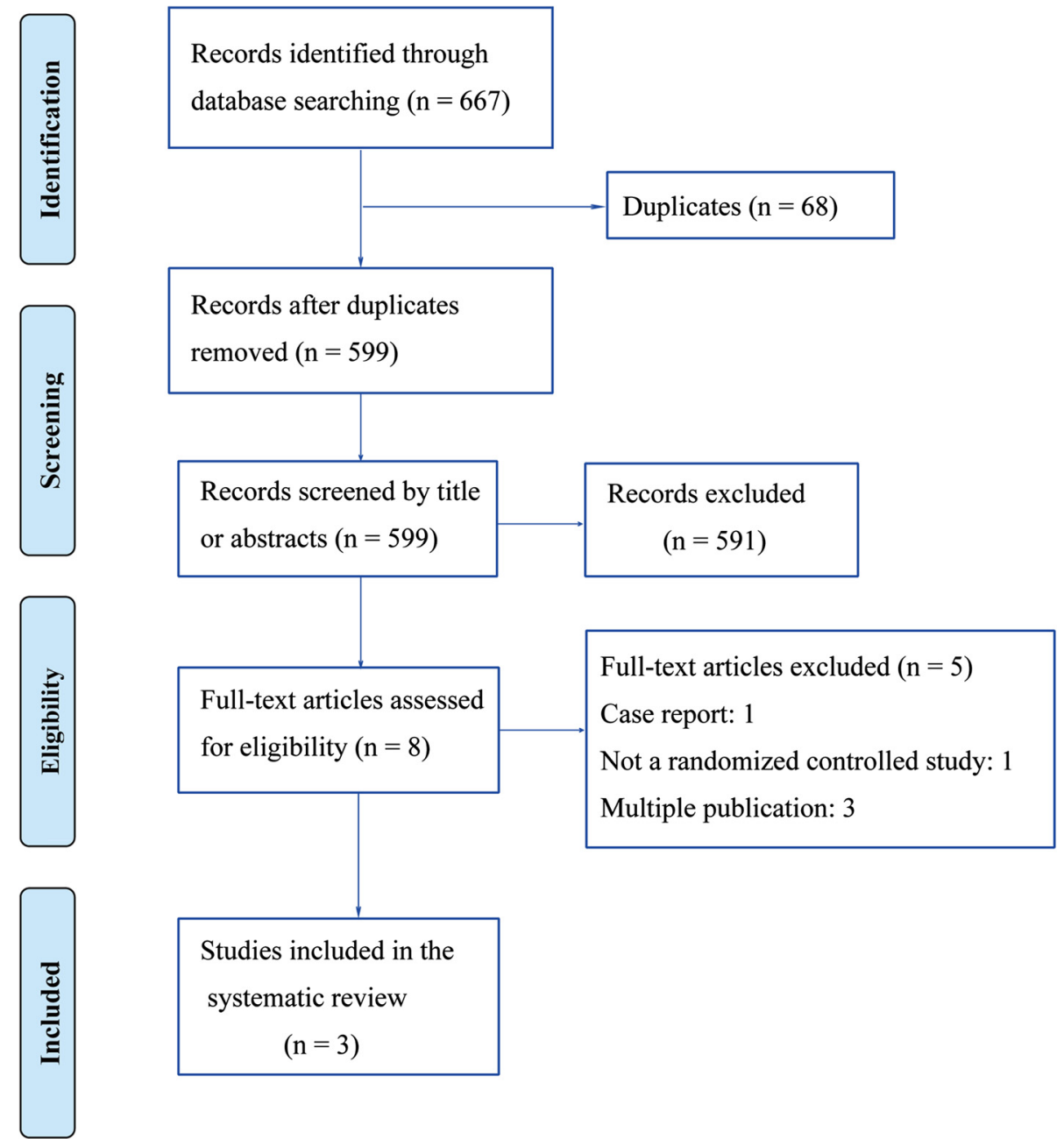

Fig. 1 Flow diagram of study selection. Doi: 10.1371/journal.pmed.1000100.g002 
Random sequence generation (selection bias)

Allocation concealment (selection bias)

Blinding of participants and personnel (performance bias)

Blinding of outcome assessment (detection bias)

Incomplete outcome data (attrition bias)

Selective reporting (reporting bias)

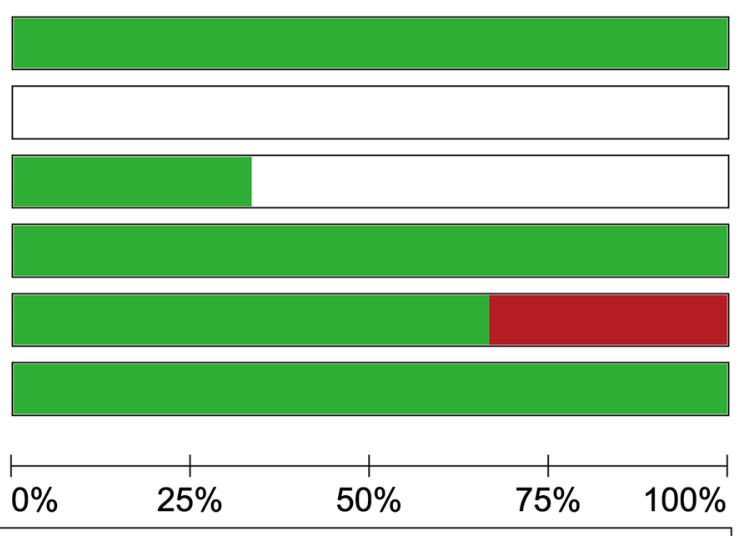

Low risk of bias

Unclear risk of bias

High risk of bias

Fig. 2 Risk of bias graph. The bias assessment items are presented as low risk, high risk, or unclear risk using different colors

(2) Same study subjects were enrolled in other reports.

(3) The SD and rTMS treatments were started in conjunction with a new antidepressant medication.

(4) The response data could not be obtained for the study even after the authors were contacted.

\section{Assessment of bias in the included studies}

To evaluate the quality of the included studies, the risk of bias assessment tool in Review Manager Software version 5.2 (Cochrane Collaboration, Oxford, England) was used to assess the random sequence generation (selection bias), allocation concealment (selection bias), blinding of participants and personnel (performance bias), blinding of outcome assessment (detection bias), incomplete outcome data (attrition bias), selective reporting (reporting bias), and other biases. The assessment of bias was performed in accordance with the relevant descriptions in the Cochrane Handbook for Systematic Reviews of Interventions [44]. If there was sufficient detail to support a judgment for each bias, they were assigned as low risk or high risk; otherwise, they were assigned as unclear risk.

\section{Data extraction}

The data were individually extracted by two reviewers: the first author and another co-author. The data were recorded as follows:

(1) Participant characteristics: including the number of subjects, mean age, gender, treatment strategy (i.e., SD + rTMS), primary diagnosis, and presence of pharmacotherapy.

(2)rTMS parameters: including stimulation frequency, intensity, sessions, total pulses, coil, stimulating position, and the method of sham rTMS.

(3) Outcome measures: HRSD scores were extracted as the primary outcome measure. The scores obtained from the other scales were defined as secondary outcome measures. Both the HRSD scores and the other scores were assessed at the endpoint of intervention and in follow-up.

(4) Adverse events: the number and type of adverse event, if reported.

\section{Review of primary outcome measures}

The primary outcome measures (HRSD scores) in the included studies were reviewed. The HRSD scores were

Table 1 Characteristics of the included studies

\begin{tabular}{|c|c|c|c|c|c|c|c|c|c|}
\hline Study & $\begin{array}{l}\text { Number of } \\
\text { subjects (Exp/Ctr) }\end{array}$ & $\begin{array}{l}\text { Mean age, } \\
Y(\text { Exp/Ctr) }\end{array}$ & $\begin{array}{l}\text { Gender } \\
\text { (F/M) }\end{array}$ & $\begin{array}{l}\text { Treatment } \\
\text { strategy }\end{array}$ & Primary diagnosis & $\begin{array}{l}\text { Diagnostic } \\
\text { criteria }\end{array}$ & $\begin{array}{l}\text { Presence of } \\
\text { pharmacotherapy }\end{array}$ & $\begin{array}{l}\text { Adverse } \\
\text { events }\end{array}$ & Dropouts \\
\hline $\begin{array}{l}\text { Eichhammer } \\
2002\end{array}$ & $20(10 / 10)$ & $46.7(44.9 / 48.5)$ & $15 / 5$ & $\begin{array}{l}\text { PSD + } \\
\text { rTMS }\end{array}$ & $\begin{array}{l}15 \text { Major Depression; } \\
5 \text { Bipolar I }\end{array}$ & DSM-IV & $\begin{array}{l}\text { Stable } \\
\text { Pharmacotherapy }\end{array}$ & - & 0 \\
\hline Kreuzer 2012 & $37(21 / 16)$ & $43.0(45.3 / 39.9)$ & 19/18 & $\begin{array}{l}\text { TSD + } \\
\text { rTMS }\end{array}$ & Acute Depression & DSM-IV & $\begin{array}{l}\text { Stable } \\
\text { Pharmacotherapy }\end{array}$ & - & 4 \\
\hline Krstić 2014 & $19(11 / 8)$ & $48.3($ (一) & $19 / 0$ & $\begin{array}{l}\text { PSD + } \\
\text { rTMS }\end{array}$ & $\begin{array}{l}\text { Unipolar Major } \\
\text { Depression }\end{array}$ & $\begin{array}{l}\text { DSM-IV } \\
\text { and MINI }\end{array}$ & $\begin{array}{l}\text { Stable } \\
\text { Pharmacotherapy }\end{array}$ & - & 0 \\
\hline
\end{tabular}

Note: $C$ tr represents control group; DSM-IV Diagnostic and Statistical Manual of Mental Disorders, 4th Edition, Exp experimental group, $F$ female, $M$ male, MINI Mini-International Neuropsychiatric Interview Criteria, PSD partial sleep deprivation, rTMS repetitive transcranial magnetic stimulation, TSD total sleep deprivation, $Y$ years; and a dash $(-)$ indicates no report in the article 
Table 2 rTMS parameters used in the included studies

\begin{tabular}{|c|c|c|c|c|c|c|c|}
\hline Study & $\begin{array}{l}\text { Frequency } \\
(\mathrm{Hz})\end{array}$ & $\begin{array}{l}\text { Intensity } \\
\text { (\% of MT) }\end{array}$ & Sessions & Total pulses & Coil/position & $\begin{array}{l}\text { Arm of sham rTMS } \\
\text { (Yes or No) }\end{array}$ & Method of sham rTMS \\
\hline Eichhammer 2002 & 10.0 & 80 & 4 & 4,000 & Figure eight-shaped / Left DLPFC & Yes & Sham Coil \\
\hline Kreuzer 2012 & 10.0 & 110 & 4 & 4,000 & Figure eight-shaped / Left DLPFC & Yes & Sham Coil \\
\hline Krstić 2014 & 1.0 & 110 & 10 & 3,000 & Figure eight-shaped / Right DLPFC & Yes & $\begin{array}{l}\text { Coil, Perpendicular } \\
\text { to the Scalp }\end{array}$ \\
\hline
\end{tabular}

Note: DLPFC represents the dorsolateral prefrontal cortex; $\mathrm{Hz}$ periods per second, $M T$ motor threshold, NR no report of the method of sham rTMS in the article, and $r$ TMS repetitive transcranial magnetic stimulation

calculated based on a multiple item questionnaire which was designed for an indication of depression, mainly including mood, feelings of guilt, suicide, insomnia, work and activities, retardation, agitation, anxiety, hypochondriasis, weight loss, somatic symptoms, and insight [45]. Data were described as mean \pm standard deviation (s.d.). If graphs were reported instead of the original data in the articles, data were extracted with the assistance of GetData Graph Digitizer 2.25 (http://getdata-graph-digitizer.com/) based on the Cochrane Handbook for Systematic Reviews of Interventions [44]. It is important to note that skewed data could not be expressed as mean \pm s.d.. They were usually reported with the means, medians, and inter-quartile ranges as well as the maximums and minimums. The authors of the included articles were contacted for more detailed information about their studies.

\section{Review of secondary outcome measures}

Apart from the HRSD score, other scales such as the CGI and a self-reported well-being scale (BfS), were used to measure the degree of depression. CGI is a 7-point scale commonly used in psychiatry, measuring the illness severity, global improvement or change, and the therapeutic response from subjective aspects, as it requires the surveyor to compare the subjects with typical patients in clinics [41]. CGI is usually used to measure depressive symptoms [46, 47]. BfS is a self-rating scale for the measurement of subjective well-being conditions. It is especially suitable to assess the rapid mood changes [48, 49]. The CGI scores and BfS scores were defined as secondary outcome measures and systematically reviewed for a supplementary proof.

\section{Results}

\section{Selection and inclusion of studies}

The original searches yielded 20 results in Embase, 29 in JSTOR, 15 in Medline, 30 in PubMed, 568 in ScienceDirect, and five in the Cochrane Central Register of Controlled Trials. After exclusion, eight potentially relevant abstracts or articles were obtained based on the title and abstract [29-34, 50]. Of these eight publications, five were excluded for the following reasons: (a) one of them was a case report [29]; (b) one was not an RCT [50]; and (c) three trials contained the same study population as other published studies $[31,34,51]$. The remaining three studies $[30,32,33]$ met the inclusion criteria described above and were therefore included in this systematic review. Skewed data were found in one study [30]. The data in the other two studies were normally distributed. The flow diagram of study selection is shown as Fig. 1.

\section{Risk of bias in the included studies}

Fig. 2 shows the risk of bias assessments for the included studies. All the trials had reported the random sequence generation, but were determined with an unclear risk of allocation concealment. Only one study reported the blinding procedure of the participants and personnel in detail [33]: "All of the patients were naive to both TMS and partial SD; and thus, it was not likely that they could recognize the treatment modality". The other two studies had unclear or high risk of bias with respect to the procedures of the participants and personnel. One study had a high risk of incomplete outcome data [32]. The blinding of outcome assessment and selective reporting in the included trials were all of low risk. No other biases had been reported in the three included studies.

\section{Basic information of the included studies \\ Characteristics of the included studies}

The characteristics of the included studies are shown in Table 1. Except for four dropouts in the active/sham rTMS treatment procedures without giving any reasons [32], 72 depressed patients with complete treatment and measurement were finally included in this systematic review. The primary diagnoses of the patients in the three studies were major depression and bipolar disorder. The patients received TSD [32] or PSD [30, 33] in combination with rTMS treatment in all of the

Table 3 Summary of the primary outcome measures

\begin{tabular}{lllcc}
\hline Study & Outcome measures & $\mathrm{N}_{\text {Exp }} / \mathrm{N}_{\text {Ctr }}$ & $\mathrm{MV}_{\text {Exp }} / \mathrm{MV}_{\text {Ctr }}$ & s.d.Exp \\
\hline Kreuzer 2012 & HRSD Scores & $19 / 14$ & $8.74 / 10.5$ & $6.09 / 5.52$ \\
Krstić 2014 & HRSD Scores & $11 / 8$ & $17.5 / 23.9$ & $5.6 / 3.8$ \\
\hline
\end{tabular}

Note: Ctr represents control group, Exp experimental group, HRSD Hamilton rating scale for depression, $M V$ mean value, $N$ number of subjects involved in the systematic review, and s.d. standard deviation 
Table 4 Summary of the secondary outcome measures

\begin{tabular}{lllcl}
\hline Study & Outcome measures & $\mathrm{N}_{\text {Exp }} / \mathrm{N}_{\text {Ctr }}$ & $\mathrm{MV}_{\text {Exp }} / \mathrm{MV}_{\text {Ctr }}$ & s.d. Exp $/$ s.d.Ctr \\
\hline Kreuzer 2012 & BfS Scores & $19 / 14$ & $23.61 / 18.71$ & $13.57 / 10.38$ \\
Krstić 2014 & CGl Scores & $11 / 8$ & $3.00 / 3.78$ & $0.89 / 0.67$
\end{tabular}

Note: BfS represents self-reported well-being scale; $C G I$ clinical global impression scale, Ctr control group, Exp experimental group, HRSD Hamilton rating scale for depression, $M V$ mean values, $N$ number of subjects involved in the systematic review, and s.d. standard deviation

studies. For TSD, the patients were deprived of sleep for a period of $24 \mathrm{~h}$ from 8:00 pm until 8:00 pm the next evening. In contrast, for PSD, the patients went to bed as usual and were woken up at 1:30 am. Then, they remained awake until 8:00 pm the next evening. All of the subjects received stable pharmacotherapy throughout the treatment process. There was no report of adverse events.

\section{rTMS parameters}

The included studies used different rTMS interventions (Table 2). One of the trials used ten sessions of LF-rTMS $(1.0 \mathrm{~Hz})$ over the right DLPFC for a total of 3,000 pulses [33], and the other two used HF-rTMS (10.0 Hz) over the left DLPFC, with four sessions of 4,000 pulses [30, 32]. The intensity varied between the different trials. Moreover, the sham rTMS control was conducted by using a sham figure eight-shaped coil $[30,32]$ or by applying a real coil perpendicular to the scalp [33].

\section{Primary outcome measures}

In Eichhammer's article [30], the data were expressed as means and a box plot diagram. In the diagram, the medians and interquartile ranges as well as the maximums and minimums were shown. From the means and the box plot diagram, it was evident that the outcome data of this trial were skewed. In their paper, Eichhammer et al. [30] reported a stabilizing antidepressant effect of rTMS for SD. The patients were designed to receive one night of PSD during four days of rTMS treatment. On the day prior to the PSD (day 0), the HRSD scores in both groups were comparable (active rTMS, $27.7 \pm 5.5$; sham rTMS, $26.9 \pm 7.3$ ). After PSD (day 1), little difference was observed between the active and sham groups (HRSD scores of $9.1 \pm 5.6$ and $9.4 \pm 4.2$, respectively). However, after completing four sessions of rTMS treatment (day 4), the mean HRSD score of the PSD responders in the active rTMS group remained at 9.0 (median, 9.0; interquartile range, $2.0 \sim 16.0$ ), while it went up to 19.0 (median, 20.0; interquartile range $13.0 \sim 24.0$ ) in the sham group.

The other two studies reported normally distributed data. Among them, Kreuzer's article [32] did not directly express the outcome measures as the mean value \pm the s.d. but provided the initial data of each subject instead. Therefore, the post-treatment mean values and s.d. of the groups were calculated based on the original data. The calculations excluded the drop-out patients, and the outcome was consistent with the line chart provided by the authors in the article. The primary outcome measures of these two studies are summarized in Table 3, including the number of subjects involved in the calculation and the mean and s.d. of the HRSD values. A bit difference was found between the two studies. In Kreuzer's article, it indeed showed a decrease of HRSD score in the experimental group (real rTMS added to SD) when compared with the control (sham rTMS added to SD), but the decrease was not significant. However, in Krstić's study, the HRSD scores in the experimental group were significantly lower than that in the control group.

\section{Secondary outcome measures}

Apart from the HRSD scores, two studies included in this systematic review also reported a secondary rating scale for the antidepressant effects of SD and rTMS. Krstić's study reported CGI scores [33]. And in Kreuzer's article, BfS scores, which were suitable for the evaluation of rapid mood changes, served as a subjective efficacy measurement [32]. The secondary outcome measures are summarized in Table 4. Both the CGI and the BfS scores showed good improvement of depressive symptoms in the experimental group.

\section{Follow-up effects}

A total of 50 subjects were enrolled in the follow-up assessment. The follow-up results of the primary and secondary outcome measures are shown in Table 5 and Table 6. The data show a maintained result with the immediate effects of the combined use of rTMS and SD.

\section{Discussion}

Although numerous systematic reviews and/or metaanalyses have been published and proved that rTMS was an effective treatment for depressive patients [52-56], there were few reviews addressing the combined therapy

Table 5 Follow-up of the primary outcome measures

\begin{tabular}{llllcc}
\hline Study & Outcome measures & Follow-up duration $(w k)$ & $N_{\text {Exp }} / \mathrm{N}_{\text {Ctr }}$ & $M_{\text {Exp }} / \mathrm{MV}_{\text {Ctr }}$ & s.d.Exp $/$ s.d.ctr \\
\hline Kreuzer 2012 & HRSD Scores & 1 & $18 / 14$ & $12.29 / 13.57$ & $8.12 / 7.65$ \\
Krstić 2014 & HRSD Scores & 3 & $10 / 8$ & $16.7 / 25.2$ & $5.7 / 4.5$ \\
\hline
\end{tabular}

Note: Ctr represents control group; Exp experimental group, HRSD Hamilton rating scale for depression, $M V$ mean values, $N$ number of subjects involved in the systematic review, s.d. standard deviation; wk, week 
Table 6 Follow-up of the secondary outcome measures

\begin{tabular}{llllcc}
\hline Study & Outcome measures & Follow-up duration $(w k)$ & $N_{\text {Exp }} / \mathrm{N}_{\text {Ctr }}$ & $\mathrm{MV}_{\text {Exp }} / \mathrm{MV}_{\text {Ctr }}$ & s.d.Exp $/$ s.d.Ctr \\
\hline Kreuzer 2012 & BfS Scores & 1 & $18 / 14$ & $25.07 / 22.33$ & $16.28 / 15.13$ \\
Krstić 2014 & CGI Scores & 3 & $10 / 8$ & $2.55 / 3.89$ & $0.93 / 0.78$ \\
\hline
\end{tabular}

Note: Ctr represents control group; Exp experimental group, HRSD Hamilton rating scale for depression, $M V$ mean values, $N$ number of subjects involved in the systematic review, s.d. standard deviation, wk week

of rTMS with other antidepressant treatments. Only one recent meta-analysis conducted by Liu et al. [57] had reported an augmentative effect of rTMS on medication treatment for medication-resistant depression patients. Nevertheless, the study of Liu et al. did not concentrate on the combined use of rTMS and SD. In this work, we reviewed the literature on the effects of SD and rTMS cotherapy for depression and attempted to determine whether rTMS could augment the antidepressive effect of SD. The publications showed different changes of depressive symptoms in the depression patients of the experimental group (SD + active rTMS) compared with the subjects of the sham control (SD + sham rTMS). The different primary outcome measures in Kreuzer's and Krstićs articles might result from the different SD strategies and rTMS parameters used in the treatment process. Currently, late PSD (in the second half of the night) has been considered to be as effective as TSD. However, doubts have also been expressed by others who consider TSD to be superior [58-60]. In Kreuzer's study [32], TSD was used prior to the sham or active rTMS. The results showed an obvious improvement in depressive symptoms following TSD, and this improvement lasted throughout the treatment period of rTMS, regardless of sham or active rTMS, which resulted in a longer-lasting antidepressant effect of TSD in the sham rTMS group. Besides, LF-rTMS and HF-rTMS might have contributed differently to depression patients. However, up to now, no other evidence has been available to differentiate the antidepressant effects between PSD and TSD, or between LF-rTMS and HF-rTMS. Therefore, further studies are needed to verify these inferences.

Depression can be associated with various factors, such as hyperglycemia [61], obesity [62], stroke [63], cardiovascular disorders, stress, and anxiety. According to Martinac's review [64], hyperactivity of the hypothalamic-pituitaryadrenal axis and changes in the immune system are characteristics of depressive disorders. Huang et al. [65] also suggested that depression-like behaviors in rodent models and in humans could be improved by enhancing the brain $\mathrm{N}$-methyl-D-aspartate function. To date, the mechanisms of the augmented effect of rTMS on SD have not been clarified. Krstić et al. concluded in their article that the combined use of rTMS and PSD or the use of either treatment alone would affect the same cortical regions with a possibly synergistic effect [34]. Moreover, the metabolic activity of the anterior cingulate cortex was considered important for the antidepressant action of both SD and rTMS, which could contribute to the enhanced efficacy of SD + rTMS co-therapy [30, 32]. Further animal model studies of depression with rTMS and SD co-therapy may help to understand the underlying mechanism.

Certain limitations exist in this systematic review. First, the outcome measures in Kreuzer's study were calculated according to the original materials provided [32], which might lead to errors with the measurements. Second, potential publication bias might be an influence factor for the power of this systematic review. Third, the included RCTs did not report the methods of allocation concealment, which induced an unclear risk of bias for the study. Finally, the present review contained only three articles from two research groups so that the total sample size was too small to accurately predict the effect of rTMS on SD. More RCTs with larger sample sizes are warranted.

\section{Conclusions}

From this systematic review, an overview of the research for the combined use of rTMS and SD is presented. Only three studies are now available in the databases. Two of them showed augmentative effects of rTMS on SD in depressive patients; while the other one showed non-significant effects. The few publications limited a conclusive evidence for the therapeutic effects of SD and rTMS co-therapy in patients with depression. However, these studies provide a direction for future research of rTMS-SD co-therapy for depression. Further well-designed studies with a larger number of patients are highly warranted to confirm whether there is an augmentative antidepressant effect of rTMS on SD.

\section{Additional file}

Additional file 1: S1 Checklist. PRISMA 2009 Checklist. (PDF 201 kb)

\section{Abbreviations}

BfS: self-reported well-being scale; CGl: clinical global impression rating scale; $\mathrm{Cl}$ : confidence intervals; Ctr: control group; DLPFC: dorsolateral prefrontal cortex; Exp: experimental group; F: female; HF-rTMS: high-frequency repetitive

transcranial magnetic stimulation; HRSD: hamilton rating scale for depression; $\mathrm{Hz}$ : periods per second; LF-rTMS: low-frequency repetitive transcranial magnetic stimulation; M: male; MADRS: Montgomery-Åsberg Depression Scale; MT: motor threshold; MV: mean values; N: number of subjects; NR: no report of the method of sham repetitive transcranial magnetic stimulation in the article; PSD: partial sleep deprivation; RCTs: randomized controlled trials; rTMS: repetitive transcranial magnetic stimulation; s.d.: standard deviation; SD: sleep deprivation; TMS: transcranial magnetic stimulation; TSD: total sleep deprivation; WMD: weighted mean differences; Y: Years. 


\section{Competing interests}

The authors declare that they have no competing interests.

\section{Authors' contributions}

QT conceived and designed the study, participated in the literature searches and drafted the manuscript. GML participated in the design of the study and the literature search. AGW conceived and designed the study and screened the articles. TL contributed to the acquisition and selection of data, and helped to draft the manuscript. SGF performed the analysis. ZWG helped to extract the data and perform the analysis. HPC contributed to the quality assessment of the included studies. BH helped to analyze the characteristics of the included studies and helped to assess the studies' quality. MAM contributed to the draft and the revision of the manuscript. JO helped to draft the manuscript. GQX revised the manuscript. QWM conceived of the study and revised the manuscript. All authors read and approved the final manuscript.

\section{Acknowledgements}

We thank Senhong Wang who provided helpful suggestions on the design of this work. This work was supported by the National Natural Science Foundation of China (NSFC, No.81271559); the State Administration of Foreign Experts Affairs, the P.R. of China (No.20155100056); the Basic Program Funded by Science \& Technology Department of Sichuan Province (No.2011HH0017); and the Scientific Research Project of Sichuan Health Department (No.110616). The sponsors had no role in the study design, in the collection, analysis and interpretation of data, in the writing of the manuscript, or in the decision to submit the manuscript for publication.

\section{Author details}

${ }^{1}$ Department of Radiology \& Imaging Institute of Rehabilitation and Development of Brain Function, North Sichuan Medical University Nanchong Central Hospital, 97 South Renmin Road, Shunqing District, Nanchong 637000, Sichuan, China. ${ }^{2}$ Department of Oncology, North Sichuan Medical University Nanchong Central Hospital, 97 South Renmin Road, Shunqing District, Nanchong 637000, Sichuan, China. ${ }^{3}$ Department of Urology Surgery, North Sichuan Medical University Nanchong Central Hospital, 97 South Renmin Road, Shunqing District, Nanchong 637000, Sichuan, China. ${ }^{4}$ Department of Cardiology, North Sichuan Medical University Nanchong Central Hospital, 97 South Renmin Road, Shunqing District, Nanchong 637000, Sichuan, China. ${ }^{5}$ Department of Nephrology, North Sichuan Medical University Nanchong Central Hospital, 97 South Renmin Road, Shunqing District, Nanchong 637000, Sichuan, China. ' Lotus Biotech.com LLC., John Hopkins University-MCC, 9601 Medical Center Drive, Rockville, MD 20850, USA. ${ }^{7}$ Peking University Third Hospital, 49 Garden North Road, Haidian District, Beijing 100080, China.

Received: 2 August 2015 Accepted: 4 November 2015 Published online: 14 November 2015

\section{References}

1. Marcus M, Yasamy MT, Ommeren M van, Chisholm D, Saxena S. Depression: A Global Public Health Concern. In: Depression. World Health Organization. 2012. http://www.who.int/mental_health/management/depression/ who_paper_depression_wfmh_2012.pdf.

2. Taghva AS, Malone DA, Rezai AR. Deep brain stimulation for treatment-resistant depression. World Neurosurg. 2013;80 Suppl 27:e17-24.

3. Roy A, Bhanji S. Sleep deprivation treatment in depression: a review. Postgrad Med J. 1976;52:50-2.

4. Hemmeter UM, Hemmeter-Spernal J, Krieg JC. Sleep deprivation in depression. Expert Rev Neurother. 2010;10:1101-15.

5. Bosch OG, Rihm JS, Scheidegger M, Landolt HP, Stämpfli P, Brakowski J, et al. Sleep deprivation increases dorsal nexus connectivity to the dorsolateral prefrontal cortex in humans. Proc Natl Acad Sci U S A. 2013;110:19597-602.

6. Novati A, Roman V, Cetin T, Hagewoud R, den Boer JA, Luiten PG, et al. Chronically restricted sleep leads to depression-like changes in neurotransmitter receptor sensitivity and neuroendocrine stress reactivity in rats. Sleep. 2008;31:1579-85.

7. Prathiba J, Kumar KB, Karanth KS. Effects of REM sleep deprivation on cholinergic receptor sensitivity and passive avoidance behavior in clomipramine model of depression. Brain Res. 2000;867:243-5.
8. Lopez-Rodriguez F, Wilson CL, Maidment NT, Poland RE, Engel J. Total sleep deprivation increases extracellular serotonin in the rat hippocampus. Neuroscience. 2003;121:523-30.

9. Benedetti F, Colombo C, Barbini B, Campori E, Smeraldi E. Ongoing lithium treatment prevents relapse after total sleep deprivation. J Clin Psychopharmacol. 1999;19:240-5.

10. Wu JC, Kelsoe JR, Schachat C, Bunney BG, DeModena A, Golshan S, et al. Rapid and sustained antidepressant response with sleep deprivation and chronotherapy in bipolar disorder. Biol Psychiatry. 2009;66:298-301.

11. Barker AT, Jalinous R, Freeston IL. Non-invasive magnetic stimulation of human motor cortex. Lancet. 1985;1:1106-7.

12. Hallett M. Transcranial magnetic stimulation and the human brain. Nature. 2000;406:147-50.

13. Pascual-Leone A, Rubio B, Pallardo F, Catala MD. Rapid-rate transcranial magnetic stimulation of left dorsolateral prefrontal cortex in drug-resistant depression. Lancet. 1996;348:233-7.

14. Eschweiler GW, Wegerer C, Schlotter W, Spandl C, Stevens A, Bartels M, et al. Left prefrontal activation predicts therapeutic effects of repetitive transcranial magnetic stimulation (rTMS) in major depression. Psychiatry Res. 2000;99:161-72.

15. Janicak PG, Dowd SM, Martis B, Alam D, Beedle D, Krasuski J, et al. Repetitive transcranial magnetic stimulation versus electroconvulsive therapy for major depression: preliminary results of a randomized trial. Biol Psychiatry. 2002;51:659-67.

16. Aarre TF, Dahl AA, Johansen JB, Kjønniksen I, Neckelmann D. Efficacy of repetitive transcranial magnetic stimulation in depression: a review of the evidence. Nord J Psychiatry. 2003;57:227-32.

17. Bares M, Kopecek M, Novak T, Stopkova P, Sos P, Kozeny J, et al. Low frequency (1-Hz), right prefrontal repetitive transcranial magnetic stimulation (rTMS) compared with venlafaxine ER in the treatment of resistant depression: a double-blind, single-centre, randomized study. J Affect Disord. 2009;118:94-100.

18. Rossini D, Lucca A, Magri L, Malaguti A, Smeraldi E, Colombo C, et al. A symptom-specific analysis of the effect of high-frequency left or low-frequency right transcranial magnetic stimulation over the dorsolateral prefrontal cortex in major depression. Neuropsychobiology. 2010;62:91-7.

19. George MS, Lisanby SH, Avery D, McDonald WM, Durkalski V, Pavlicova M, et al. Daily left prefrontal transcranial magnetic stimulation therapy for major depressive disorder: a sham-controlled randomized trial. Arch Gen Psychiatry. 2010;67:507-16.

20. Krisanaprakornkit T, Paholpak S, Tassaniyom K, Pimpanit V. Transcranial magnetic stimulation for treatment resistant depression: six case reports and review. J Med Assoc Thai. 2010;93:580-6.

21. Schlaepfer TE, Kosel M, Fisch HU. Repetitive transcranial magnetic stimulation (rTMS) in depression. Poiesis Prax. 2006:4:111-27.

22. Baeken C, Vanderhasselt MA, Remue J, Herremans S, Vanderbruggen N, Zeeuws $D$, et al. Intensive HF-rTMS treatment in refractory medicationresistant unipolar depressed patients. J Affect Disord. 2013;151:625-31.

23. George MS, Taylor JJ, Short EB. The expanding evidence base for rTMS treatment of depression. Curr Opin Psychiatry. 2013;26:13-8.

24. Baeken C, Marinazzo D, Wu GR, Van Schuerbeek P, De Mey J, Marchetti I, et al. Accelerated HF-rTMS in treatment-resistant unipolar depression: Insights from subgenual anterior cingulate functional connectivity. World J Biol Psychiatry. 2014;15:286-97.

25. Takahashi S, Ukai S, Tsuji T, Kose A, Shoyama M, Yamamoto M, et al Cerebral blood flow in the subgenual anterior cingulate cortex and modulation of the mood-regulatory networks in a successful rTMS treatment for major depressive disorder. Neurocase. 2013;19:262-7.

26. Dougherty DD, Rauch SL. Somatic therapies for treatment-resistant depression: new neurotherapeutic interventions. Psychiatr Clin North Am. 2007;30:31-7.

27. Mobascher A, Arends M, Eschweiler GW, Brinkmeyer J, Agelink MW, Kornischka J, et al. Biological correlates of prefrontal activating and temporoparietal inhibiting treatment with repetitive transcranial magnetic stimulation (rTMS). Fortschr Neurol Psychiatr. 2009;77:432-43.

28. Choi KM, Jang KM, Jang Kl, Um YH, Kim MS, Kim DW, et al. The effects of 3 weeks of rTMS treatment on P200 amplitude in patients with depression. Neurosci Lett. 2014;577:22-7.

29. Krstić J, Ilić TV. Switch to hypomania induced by repetitive transcranial magnetic stimulation and partial sleep deprivation added to antidepressant: A case report. Vojnosanit Pregl. 2014;71:207-10.

30. Eichhammer P, Kharraz A, Wiegand R, Langguth B, Frick U, Aigner JM, et al. Sleep deprivation in depression: Stabilizing antidepressant effects by repetitive transcranial magnetic stimulation. Life Sci. 2002;70:1741-9. 
31. Nic TV, Krstic J, Diligenski V. Slow-rate tonic rTMS combined with sleep deprivation in treatment of pharmacoresistant depression. Clin Neurophysiol. 2010;121:S167.

32. Kreuzer PM, Langguth $B$, Schecklmann M, Eichhammer $P$, Hajak G, Landgrebe $M$. Can repetitive transcranial magnetic stimulation prolong the antidepressant effects of sleep deprivation. Brain Stimul. 2012;5:141-7.

33. Krstić J, Buzadžić I, Milanović SD, Ilić NV, Pajić S, Ilić TV. Low-Frequency repetitive transcranial magnetic stimulation in the right prefrontal cortex combined with partial sleep deprivation in treatment-resistant depression: a randomized sham-controlled trial. J ECT. 2014;30:325-31.

34. Krstić J, Diligenski V, llić TV. Low-frequency repetitive transcranial magnetic stimulation conjugated with partial sleep deprivation in treatment of major depression - pilot study. Clin Neurophysiol. 2010;121:e5-18.

35. Bermingham EN, Thomas DG, Cave NJ, Morris PJ, Butterwick RF, German AJ. Energy requirements of adult dogs: a meta-analysis. PLoS One. 2014;9: e109681.

36. Moher D, Liberati A, Tetzlaff J, Altman DG. Preferred reporting items for systematic reviews and meta-analyses: the PRISMA statement. PLoS Med. 2009;6:e1000097.

37. Liberati A, Altman DG, Tetzlaff J, Mulrow C, Gotzsche PC, Loannidis JP, et al. The PRISMA statement for reporting systematic reviews and meta-analyses of studies that evaluate health care interventions: explanation and elaboration. PLoS Med. 2009;6:e1000100.

38. Berlim MT, van den Eynde F, Tovar-Perdomo S, Daskalakis ZJ. Response, remission and drop-out rates following high-frequency repetitive transcranial magnetic stimulation (rTMS) for treating major depression: a systematic review and meta-analysis of randomized, double-blind and sham-controlled trials. Psychol Med. 2014;44:225-39.

39. American Psychiatric Association. Diagnostic and statistical manual of mental disorders. 4th ed. Washington: American Psychiatric Association; 1994.

40. Sheehan DV, Lecrubier Y, Sheehan KH, Amorim P, Janavs J, Weiller E, et al. The Mini-International Neuropsychiatric Interview (M.I.N.I.): the development and validation of a structured diagnostic psychiatric interview for DSM-IV and ICD-10. J Clin Psychiatry. 1998;59 Suppl 20:22-33.

41. Hamilton M. A rating scale for depression. J Neurosurg Psychiatry. 1960;23:56-62.

42. Guy W. ECDEU Assessment Manual for Psychopharmacology. Bethesda: U.S. Department of Health, Education and Welfare publication; 1976.

43. Montgomery SA, Åsberg M. A new depression scale designed to be sensitive to change. Br J Psychiatry. 1979;134:382-9.

44. Higgins J, Green S (editors). Cochrane Handbook for Systematic Reviews of Interventions Version 5.1.0. In: The Cochrane Collaboration. 2011. http:// www.cochrane-handbook.org.

45. Hamilton M. Development of rating scale for primary depressive illness. Br J Soc Clin Psychol. 1967;6:278-96.

46. Rocha FL, Hara C. Lamotrigine augmentation in unipolar depression. Int Clin Psychopharmacol. 2003;18:97-9.

47. Geddes JR, Freemantle N, Mason J, Eccles MP, Boynton J. WITHDRAWN: Selective serotonin reuptake inhibitors (SSRIS) versus other antidepressants for depression. Cochrane Database Syst Rev. 2007;18:CD001851.

48. Schwarz D, Strian F. Psychometric studies on well-being in psychiatric and medical patients. Arch Psychiatr Nervenkr. 1972;216:70-81.

49. Joseph S, Linley PA, Harwood J, Lewis CA, McCollam P. Rapid assessment of well-being: The Short Depression-Happiness Scale (SDHS). Psychol Psychother. 2004;77:463-78.

50. Padberg F, Schüle C, Zwanzger P, Baghai T, Ella R, Mikhaiel P, et al. Relation between responses to repetitive transcranial magnetic stimulation and partial sleep deprivation in major depression. J Psychiatr Res. 2002;36:131-5.

51. Wiegand R, Eichhammer P, Langguth B, Kharras A, Hajak G. Sleep deprivation in depression: stabilizing antidepressant effects by rtms. Int J Neuropsychoph. 2002;5 Suppl 1:202.

52. Gross M, Nakamura L, Pascual-Leone A, Fregni F. Has repetitive transcranial magnetic stimulation (rTMS) treatment for depression improved? A systematic review and meta-analysis comparing the recent vs. the earlier rTMS studies. Acta Psychiatr Scand. 2007;116:165-73.

53. Lam RW, Chan P, Wilkins-Ho M, Yatham LN. Repetitive transcranial magnetic stimulation for treatment-resistant depression: a systematic review and metaanalysis. Can J Psychiatry. 2008;53:621-31.

54. Schutter DJ. Antidepressant efficacy of high-frequency transcranial magnetic stimulation over the left dorsolateral prefrontal cortex in double-blind sham-controlled designs: a meta-analysis. Psychol Med. 2009;39:65-75.
55. Chen J, Zhou C, Wu B, Wang Y, Li Q, Wei Y, et al. Left versus right repetitive transcranial magnetic stimulation in treating major depression: a meta-analysis of randomized controlled trials. Psychiatry Res. 2013;210:1260-4.

56. Gaynes BN, Lloyd SW, Lux L, Gartlehner G, Hansen RA, Brode S, et al. Repetitive transcranial magnetic stimulation for treatment-resistant depression: a systematic review and meta-analysis. J Clin Psychiatry. 2014;75:477-89.

57. Liu B, Zhang Y, Zhang L, Li L. Repetitive transcranial magnetic stimulation as an augmentative strategy for treatment-resistant depression, a meta-analysis of randomized, double-blind and sham-controlled study. BMC Psychiatry. 2014;14:342.

58. Wehr T. Effects of wakefulness and sleep on depression and mania. In: Mountplaisir J, Godbout R, editors. Sleep and biological rhythms: Basic mechanisms and applications to psychiatry. Oxford: Oxford University Press; 1990. p. 42-86.

59. Giedke H, Klingberg S, Schwärzler F, Schweinsberg M. Direct comparison of total sleep deprivation and late partial sleep deprivation in the treatment of major depression. J Affect Disord. 2003;76:85-93.

60. Giedke H, Schwärzler F. Therapeutic use of sleep deprivation in depression. Sleep Med Rev. 2002;6:361-77.

61. Wang Z, Luo W, Li P, Qiu J, Luo Q. Acute hyperglycemia comprises cerebral blood flow following cortical spreading depression in rats monitored by laser speckle imaging. J Biomed Opt. 2008;13:064023.

62. Hung CF, Rivera M, Craddock N, Owen MJ, Gill M, Korszun A, et al. Relationship between obesity and the risk of clinically significant depression: Mendelian randomization study. Br J Psychiatry. 2014;205:24-8.

63. Kunze A, Zierath D, Drogomiretskiy O, Becker K. Strain differences in fatigue and depression after experimental stroke. Transl Stroke Res. 2014;5:604-11.

64. Martinac M, Pehar D, Karlović D, Babić D, Marcinko D, Jakovljević M. Metabolic syndrome, activity of the hypothalamic-pituitary-adrenal axis and inflammatory mediators in depressive disorder. Acta Clin Croat. 2014;53:55-71.

65. Huang CC, Wei IH, Huang CL, Chen KT, Tsai MH, Tsai P, et al. Inhibition of glycine transporter-I as a novel mechanism for the treatment of depression. Biol Psychiatry. 2013;74:734-41.

\section{Submit your next manuscript to BioMed Central and take full advantage of:}

- Convenient online submission

- Thorough peer review

- No space constraints or color figure charges

- Immediate publication on acceptance

- Inclusion in PubMed, CAS, Scopus and Google Scholar

- Research which is freely available for redistribution 